Hina M. Dutt • M. Safdar • Asghar Qadir

\title{
Linearization criteria for two-dimensional systems of third-order ordinary differential equations by complex approach
}

Received: 22 November 2018 / Accepted: 10 January 2019 / Published online: 28 January 2019 (C) The Author(s) 2019

\begin{abstract}
Linearization criteria for two-dimensional systems of second-order ordinary differential equations (ODEs) have been derived earlier using complex symmetry analysis. For such systems, the linearizable form, linearization criteria and symmetry group classification are presented. In this paper, we extend the complex approach to obtain a complex-linearizable form of two-dimensional systems of third-order ODEs. This form leads to a linearizable class and linearization criteria of these systems of ODEs.
\end{abstract}

Mathematics Subject Classification $34 \mathrm{~A} 05 \cdot 35 \mathrm{~A} 24 \cdot 76 \mathrm{M} 60 \cdot 93 \mathrm{C} 15$

\section{Introduction}

There are many methods available to solve differential equations analytically, except for some special cases of nonlinear ones that do not lend themselves to analytic solution. Those special cases often rely on transformations of the dependent and independent variables so as to cast the nonlinear equations into linear forms. One can then solve the transformed linear equations and by the inverse transformation obtain solutions of the nonlinear equations. This procedure was formalized and developed by Sophus Lie, who extended the Galois idea of using symmetries of the roots of polynomial equations to differentiable equations. Whereas Galois had used finite groups Lie required continuously infinite differentiable groups, in particular, one parameter Lie groups of transformations which are now called Lie groups [10]. Such transformations are called point transformations. It turned out that one did not need to convert to linear form to use symmetry methods to solve the equations. However, it is obviously more convenient if they can be so cast. Lie demonstrated that a scalar second-order ordinary differential equation (ODE) can be linearized if and only if there exist eight linearly independent infinitesimal symmetry generators [11].

\section{H. M. Dutt}

Department of Basic Sciences, School of Electrical Engineering and Computer Science, Islamabad, Pakistan

E-mail: hina.dutt@ seecs.edu.pk

M. Safdar

School of Mechanical and Manufacturing Engineering, Islamabad, Pakistan

E-mail: safdar.camp@gmail.com

\section{A. Qadir}

Physics Department, School of Natural Sciences, Islamabad, Pakistan

H. M. Dutt · M. Safdar · A. Qadir $(\square)$

National University of Sciences and Technology, Campus H-12, Islamabad 44000, Pakistan

E-mail: asgharqadir46@gmail.com 
Though there is a unique class of linearizable second-order scalar ODEs, the same cannot be said for $n$th $(n>2)$ order scalar ODEs or $m$-dimensional $(m \geq 2)$ systems of $n$th $(n \geq 2)$ order ODEs. Mahomed and Leach [12] showed that for $n$ th-order linearizable ODEs, for $n \geq 3$, there are three classes with $n+1, n+2$ or $n+4$ symmetry generators. For $m$-dimensional systems of second-order ODEs, there are $m+3$ classes, for example in case of two-dimensional linearizable systems of second-order ODEs there are five classes with $5,6,7,8$ or 15 symmetry generators. However, there is no classification available for linearizable systems of $n$ th-order $(n>3)$ ODEs. As such, any progress towards canonically linearizing third-order systems is very useful, even if it is not a fully general canonical form of these systems of ODEs. In this paper, we present such a procedure for linearization of two-dimensional systems of third-order ODEs, based on complex methods for Lie symmetry analysis.

The plan of the paper is as follows. In the next section, a very quick review of linearization of second and higher order scalar ODEs by point transformations and of complex symmetry analysis is given. In the third section, complex methods are used to obtain complex linearizable two-dimensional systems of thirdorder ODEs that are then linearized. Examples are provided in fourth section. The subsequent section is on conclusion.

\section{Preliminaries}

Scalar first-order ODEs can always be linearized by point transformations. However, scalar second-order ODEs are linearizable if they are at most cubic in the first derivative of the dependent variable, $u(x)$,

$$
u^{\prime \prime}=A(x, u) u^{\prime 3}+B(x, u) u^{\prime 2}+C(x, u) u^{\prime}+D(x, u)=0,
$$

where $A, B, C, D$ satisfy (see, e.g [13]) a set of four first-order constraints containing these coefficients and two auxiliary functions. Tressè [20] reduced them to two second-order conditions

$$
\begin{aligned}
& 3 A_{x x}+3 A_{x} C-3 A_{u} D+3 A C_{x}+C_{u u}-6 A D_{u}+B C_{u}-2 B B_{x}-2 B_{x u}=0, \\
& 6 A_{x} D-3 B_{u} D+3 A D_{x}+B_{x x}-2 C_{x u}-3 B D_{u}+3 D_{u u}+2 C C_{u}-B_{x} C=0,
\end{aligned}
$$

where $x$ and $u$ in the subscripts denote partial derivatives with respect to these variables. We call (1) Lie linearizable. Chern [5] and Grebot [7] considered scalar third-order ODEs to derive linearizability criteria and investigate their geometry. To be Lie linearizable scalar second-order nonlinear ODEs must have eight Lie point symmetries. For scalar linearizable ODEs of order $n \geq 3$, it was proved that there are three equivalence classes with $n+1, n+2$ or $n+4$ infinitesimal symmetry generators [12]. Neut and Petitot [16] considered general third-order ODEs to provide linearization criteria. Ibragimov and Meleshko (IM) [8] used the Lie procedure [11] of point transformations to determine Lie linearizability criteria for third-order ODEs. They showed that any third-order ODE $u^{\prime \prime \prime}=f\left(x ; u, u^{\prime}, u^{\prime \prime}\right)$, obtained from a linear equation

$$
s^{\prime \prime \prime}(t)+\kappa(t) s(t)=0,
$$

by means of point transformations

$$
t=\varphi(x, u), \quad s=\psi(x, u),
$$

must belong to one of the following two types of equations.

Type I: If $\varphi_{u}=0$, i.e.

$$
t=\varphi(x), \quad s=\psi(x, u),
$$

then the Lie linearizable form of the third-order ODE is

$$
u^{\prime \prime \prime}+\left(a_{1} u^{\prime}+a_{0}\right) u^{\prime \prime}+b_{3} u^{\prime 3}+b_{2} u^{\prime 2}+b_{1} u^{\prime}+b_{0}=0,
$$

where the coefficients $a_{j}, b_{k}$ for $j=0,1, k=0,1,2,3$, are functions of $x$ and $u$. Equation (6) is reducible to (3) if $a_{j}, b_{k}$ satisfy the following conditions: 


$$
\begin{aligned}
& a_{0 u}-a_{1 x}=0, \quad\left(3 b_{1}-a_{0}^{2}-3 a_{0 x}\right)_{u}=0, \\
& 3 a_{1 x}+a_{0} a_{1}-3 b_{2}=0, \quad 3 a_{1 u}+a_{1}^{2}-9 b_{3}=0, \\
& \left(9 b_{1}-6 a_{0 x}-2 a_{0}^{2}\right) a_{1 x}+9\left(b_{1 x}-a_{1} b_{0}\right)_{u}+3 b_{1 u} a_{0}-27 b_{0 u и}=0,
\end{aligned}
$$

where $u$ and $x$ in the subscripts denote partial derivatives.

Type II: If $\varphi_{u} \neq 0$, setting $\lambda(x, u)=\varphi_{x} / \varphi_{u}$, leads us to the following linearizable form

$$
\begin{aligned}
& u^{\prime \prime \prime}+\frac{1}{u^{\prime}+\lambda}\left[-3\left(u^{\prime \prime}\right)^{2}+\left(c_{2} u^{\prime 2}+c_{1} u^{\prime}+c_{0}\right) u^{\prime \prime}+d_{5} u^{\prime 5}\right. \\
& \left.+d_{4} u^{\prime 4}+d_{3} u^{\prime 3}+d_{2} u^{\prime 2}+d_{1} u^{\prime}+d_{0}\right]=0,
\end{aligned}
$$

where all coefficients $c_{l}, d_{m}$ for $l=0,1,2$ and $m=0,1, \ldots, 5$, are functions of $x$ and $u$. Third-order ODEs of the form (8) are reducible to (3) subject to the linearization constraint requirements given in [8]. Subsequently, Ibragimov, Meleshko and Suksern [9,19] used point and contact transformations to determine criteria for linearizability of scalar fourth-order ODEs.

In [4], linearization criteria for a two-dimensional system of second-order ODEs under general point transformations were obtained. The necessary and sufficient conditions for a system of second-order ODEs to be equivalent to a linear system with constant coefficients via fibre preserving point transformations were provided in [17]. Similarly, a few other approaches, e.g. geometric and algebraic methods, etc. have been adopted to linearize two-dimensional systems of second-order ODEs [14,15,18,21].

Considering the dependent variable as a complex analytic function of a complex independent variable and writing them in terms of their real and imaginary parts with the Cauchy-Riemann (CR) equations for analyticity, we could write a scalar ODE of any order as a pair of PDEs of the same order, subject to the two (CR) first-order constraints. The symmetries of the resulting system are different from the symmetries of the original ODE. Lie symmetry analysis using this complex splitting has been called complex symmetry analysis (CSA) [1,2]. Taking $x$ to be real and $u(x)=y_{1}(x)+i y_{2}(x)$, for an $n$th order scalar ODE

$$
u^{(n)}=\omega\left(x ; u, u^{\prime}, u^{\prime \prime}, \ldots, u^{(n-1)}\right)
$$

we obtain the two-dimensional system of ODEs

$$
\begin{aligned}
y_{1}^{(n)} & =\omega_{1}\left(x ; y_{1}, y_{2}, y_{1}^{\prime}, y_{2}^{\prime}, \ldots, y_{1}^{(n-1)}, y_{2}^{(n-1)}\right), \\
y_{2}^{(n)} & =\omega_{2}\left(x ; y_{1}, y_{2}, y_{1}^{\prime}, y_{2}^{\prime}, \ldots, y_{1}^{(n-1)}, y_{2}^{(n-1)}\right) .
\end{aligned}
$$

Linearizability of such systems implies linearizability of the complex scalar equation constructed from it; the converse is not true; linearizability of the complex scalar equation does not imply linearizability of the system [3]. If the scalar ODE is linearizable and the resulting system is also linearizable, this procedure is called complex linearization. The complex-linearization criteria for two-dimensional system of second-order ODEs have been constructed [6]. The major role in the construction is of the real linearizing transformations that must be of the form

$$
\hat{x}=\phi(x), \quad \hat{y}_{p}=\psi_{p}\left(x ; y_{1}, y_{2}, \ldots, y_{n}\right), \quad p=1,2, \ldots, n,
$$

where the $\psi_{j}$ satisfy the CR conditions. Linearizability of two-dimensional systems of second-order ODEs cannot be achieved by CSA if the linearizing point transformations are more general than (11). Here, we use these fibre preserving transformations with CR structure to obtain the linearizable form and linearization criteria for two-dimensional systems of third-order ODEs. We exploit complex-linearization for studying linearization of systems of third-order ODEs, as it provides us means to address those linearization problems which cannot be dealt with using classical symmetry analysis. Moreover, derivation of the linearization criteria becomes complicated with an increase in the order of ODEs and dimensions of their systems. In this paper, complex linearization criteria are derived which led to linearization of a class of systems of third-order ODEs that can be linearized without the usual cumbersome calculations associated. 


\section{Linearization of two-dimensional systems of third-order ODEs}

Not all two-dimensional systems of ODEs will correspond to complex scalar equations as the system must also possess the CR structure. We will use the splitting procedure for the scalar third-order ODE (6). Splitting the coefficients in that equation as:

$$
\begin{aligned}
& a_{j}(x, u)=\alpha_{j}(x, y, z)+i \beta_{j}(x, y, z) ; \quad(j=0,1), \\
& b_{k}(x, u)=\gamma_{k}(x, y, z)+i \delta_{k}(x, y, z) ; \quad(k=0,1,2,3),
\end{aligned}
$$

converts it to a system of two third-order ODEs. Here, we consider linearization of those systems that are obtainable from (6). Using $u(x)=y(x)+i z(x)$ and (12) in (6) yields the following system:

$$
\begin{gathered}
y^{\prime \prime \prime}+\left(\alpha_{1} y^{\prime}-\beta_{1} z^{\prime}+\alpha_{0}\right) y^{\prime \prime}-\left(\beta_{1} y^{\prime}+\alpha_{1} z^{\prime}+\beta_{0}\right) z^{\prime \prime}+\gamma_{3} y^{3}-3 \delta_{3} y^{\prime 2} z^{\prime} \\
-3 \gamma_{3} y^{\prime} z^{\prime 2}+\delta_{3} z^{\prime 3}+\gamma_{2} y^{\prime 2}-2 \delta_{2} y^{\prime} z^{\prime}-\gamma_{2} z^{\prime 2}+\gamma_{1} y^{\prime}-\delta_{1} z^{\prime}+\gamma_{0}=0, \\
z^{\prime \prime \prime}+\left(\beta_{1} y^{\prime}+\alpha_{1} z^{\prime}+\beta_{0}\right) y^{\prime \prime}+\left(\alpha_{1} y^{\prime}-\beta_{1} z^{\prime}+\alpha_{0}\right) z^{\prime \prime}+\delta_{3} y^{\prime 3}+3 \gamma_{3} y^{\prime 2} z^{\prime} \\
-3 \delta_{3} y^{\prime} z^{\prime 2}-\gamma_{3} z^{\prime 3}+\delta_{2} y^{\prime 2}+2 \gamma_{2} y^{\prime} z^{\prime}-\delta_{2} z^{\prime 2}+\delta_{1} y^{\prime}+\gamma_{1} z^{\prime}+\delta_{0}=0,
\end{gathered}
$$

where the coefficients $\alpha_{j}, \beta_{j} ;(j=0,1), \gamma_{k}, \delta_{k} ;(k=0,1,2,3)$, satisfy the following CR-equations

$$
\begin{aligned}
& \alpha_{j y}=\beta_{j z}, \quad \alpha_{j z}=-\beta_{j y} ; \quad(j=0,1) \\
& \gamma_{k y}=\delta_{k z}, \quad \gamma_{k z}=-\delta_{k y} ; \quad(k=0,1,2,3) .
\end{aligned}
$$

This procedure leads to the following theorems.

Theorem 1 The system of ODEs (13) with the CR equations (14) represents the most general form of twodimensional systems of third-order ODEs that can be a candidate of linearization due to complex symmetry analysis.

Proof This result follows from [6], that is, by incorporating point transformations

$$
t=\varphi(x), \quad v=\psi_{1}(x, y, z), \quad w=\psi_{2}(x, y, z)
$$

with the CR structure

$$
\psi_{1 y}=\psi_{2 z}, \quad \psi_{1 z}=-\psi_{2 y}
$$

in the system

$$
\begin{aligned}
& v^{\prime \prime \prime}(t)+\kappa_{1}(t) v(t)-\kappa_{2}(t) w(t)=0, \\
& w^{\prime \prime \prime}(t)+\kappa_{2}(t) v(t)+\kappa_{1}(t) w(t)=0,
\end{aligned}
$$

that corresponds to (3) when $s(t)=v(t)+i w(t)$ and $\kappa=\kappa_{1}(t)+i \kappa_{2}(t)$, we obtain the most general complex linearizable form (13).

Theorem 2 The sufficient conditions for a two-dimensional system of third-order ODEs of the form (13) to be linearizable are: the coefficients satisfy CR equations (14) and the following conditions

$$
\begin{aligned}
& \alpha_{0 y}+\beta_{0 z}-2 \alpha_{1 x}=0, \quad \beta_{0 y}-\alpha_{0 z}-2 \beta_{1 x}=0 \\
& 3 \alpha_{1 x}+\alpha_{0} \alpha_{1}-\beta_{0} \beta_{1}-3 \gamma_{2}=0, \quad 3 \beta_{1 x}+\alpha_{0} \beta_{1}+\alpha_{1} \beta_{0}-3 \delta_{2}=0 \\
& 3 \alpha_{1 y}+3 \beta_{1 z}+2 \alpha_{1}^{2}-2 \beta_{1}^{2}-18 \gamma_{3}=0, \quad 3 \beta_{1 y}-3 \alpha_{1 z}+4 \alpha_{1} \beta_{1}-18 \delta_{3}=0 \\
& 3 \gamma_{1 y}+3 \delta_{1 z}-2\left(\alpha_{0} \beta_{0}\right)_{z}-\left(\alpha_{0}\right)^{2}{ }_{y}+\left(\beta_{0}\right)^{2}{ }_{y}-3 \beta_{0 x z}-3 \alpha_{0 x y}=0 \\
& 3 \delta_{1 y}-3 \gamma_{1 z}-2\left(\alpha_{0} \beta_{0}\right)_{y}+\left(\alpha_{0}\right)^{2} z-\left(\beta_{0}\right)^{2}-3 \beta_{0 x y}+3 \alpha_{0 x z}=0 \\
& 4\left(9 \gamma_{1}-6 \alpha_{0 x}-2 \alpha_{0}^{2}+2 \beta_{0}^{2}\right) \alpha_{1 x}-4\left(9 \delta_{1}-6 \beta_{0 x}-4 \alpha_{0} \beta_{0}\right) \beta_{1 x}+18 \gamma_{1 x y}+18 \delta_{1 x z}-18\left(\alpha_{1} \gamma_{0}\right)_{y} \\
& \quad+18\left(\beta_{1} \delta_{0}\right)_{y}-18\left(\alpha_{1} \delta_{0}\right)_{z}-18\left(\beta_{1} \gamma_{0}\right)_{z}+6\left(\gamma_{1 y} \alpha_{0}+\delta_{1 z} \alpha_{0}+\gamma_{1 z} \beta_{0}-\delta_{1 y} \beta_{0}\right) \\
& \quad-27\left(\gamma_{0 y y}+2 \delta_{0 y z}-\gamma_{0 z z}\right)=0 \\
& 4\left(9 \gamma_{1}-6 \alpha_{0 x}-2 \alpha_{0}^{2}+2 \beta_{0}^{2}\right) \beta_{1 x}+4\left(9 \delta_{1}-6 \beta_{0 x}-4 \alpha_{0} \beta_{0}\right) \alpha_{1 x}-18 \gamma_{1 x z}+18 \delta_{1 x y}+18\left(\alpha_{1} \gamma_{0}\right)_{z} \\
& \quad-18\left(\beta_{1} \delta_{0}\right)_{z}-18\left(\alpha_{1} \delta_{0}\right)_{y}-18\left(\beta_{1} \gamma_{0}\right)_{y}+6\left(\gamma_{1 y} \beta_{0}+\delta_{1 z} \beta_{0}-\gamma_{1 z} \alpha_{0}+\delta_{1 y} \alpha_{0}\right) \\
& \quad-27\left(\delta_{0 y y}-2 \gamma_{0 z y}-\delta_{0 z z}\right)=0 .
\end{aligned}
$$


Proof Linearization criteria for two-dimensional systems of second-order ODEs with CR structure, by point transformations of the form (15) along with the CR constraints (16), were derived in [6], where it was shown that splitting the criteria for the base complex equations due to complex transformations (5) into the real and imaginary parts provides the sufficient conditions for linearization of the corresponding systems. Similarly, linearization criteria for two-dimensional systems of third-order ODEs (13) along with (14) are provided by splitting the linearization criteria (7) for the scalar third-order ODEs.

The scalar third-order ODEs of the form (8) and their associated linearization criteria cannot be extended to linearization criteria of the corresponding system of third-order equations. This class of scalar third-order ODEs (8) has been derived by exploiting general point transformations (4) that leads to complex linearization instead of linearization [6]. The linearizing conditions associated with this class of scalar equations reveal complex linearizability of the corresponding systems rather than ensuring there transformation to linear forms. However, complex linearizability of systems ensures linearization of the base complex equations.

\section{Applications}

In this section, we present applications of the theory developed, i.e. demonstrate linearization of twodimensional systems of third-order ODEs of the form (13) using complex symmetry analysis approach. The examples are for illustrative purposes to show how the conditions are checked and the solution obtained, and are not obtained from applications elsewhere.

Example 1 Consider the two-dimensional system of nonlinear third-order ODEs

$$
\begin{gathered}
y^{\prime \prime \prime}+\left(3 y^{\prime}+\frac{3}{x}\right) y^{\prime \prime}-3 z^{\prime} z^{\prime \prime}+y^{\prime 3}-3 y^{\prime} z^{2}+\frac{3}{x} y^{\prime 2}-\frac{3}{x} z^{\prime 2}=0, \\
z^{\prime \prime \prime}+3 z^{\prime} y^{\prime \prime}+\left(3 y^{\prime}+\frac{3}{x}\right) z^{\prime \prime}+3 y^{\prime 2} z^{\prime}-z^{\prime 3}+\frac{6}{x} y^{\prime} z^{\prime}=0 .
\end{gathered}
$$

The non-zero coefficients are

$$
\alpha_{1}=3, \quad \alpha_{0}=\frac{3}{x}, \quad \gamma_{3}=\delta_{3}=1, \quad \gamma_{2}=\frac{3}{x} .
$$

The above system is of the form (13) and its coefficients satisfy the CR equations and the constraints (18). Hence, it is linearizable and transforms to

$$
v^{\prime \prime \prime}=0, \quad w^{\prime \prime \prime}=0,
$$

where prime denotes differentiation with respect to $t$, under an invertible transformation of the independent and dependent variables

$$
t=x, \quad v=x e^{y} \cos z, \quad w=x e^{y} \sin z
$$

The solution of (20) is

$$
v=c_{1} t^{2}+c_{2} t+c_{3}, \quad w=c_{4} t^{2}+c_{5} t+c_{6}
$$

where all $c_{1} \ldots, c_{6}$ are arbitrary constants. Using transformation (21), we obtain the general solution

$$
y=\frac{1}{2} \ln \left[\left(c_{1} x+c_{2}+\frac{c_{3}}{x}\right)^{2}+\left(c_{4} x+c_{5}+\frac{c_{6}}{x}\right)^{2}\right], \quad z=\arctan \left(\frac{c_{4} x^{2}+c_{5} x+c_{6}}{c_{1} x^{2}+c_{2} x+c_{3}}\right),
$$

of the nonlinear system (19).

Example 2 The two-dimensional system of nonlinear third-order ODEs

$$
\begin{aligned}
& y^{\prime \prime \prime}+\left(\frac{3 y^{\prime} y^{\prime \prime}-3 z^{\prime} z^{\prime \prime}}{y^{2}+z^{2}}\right) y+\left(\frac{3 z^{\prime} y^{\prime \prime}+3 y^{\prime} z^{\prime \prime}}{y^{2}+z^{2}}\right) z=0, \\
& z^{\prime \prime \prime}+\left(\frac{3 z^{\prime} y^{\prime \prime}+3 y^{\prime} z^{\prime \prime}}{y^{2}+z^{2}}\right) y-\left(\frac{3 y^{\prime} z^{\prime \prime}-3 z^{\prime} y^{\prime \prime}}{y^{2}+z^{2}}\right) z=0,
\end{aligned}
$$


has the same form as given in (13). Moreover, its non-zero coefficients

$$
\alpha_{1}=\frac{3 y}{y^{2}+z^{2}}, \quad \beta_{1}=\frac{-3 z}{y^{2}+z^{2}},
$$

satisfy the requirements of Theorem 2. So this system is transformable to a linear system

$$
v^{\prime \prime \prime}=0, \quad w^{\prime \prime \prime}=0 .
$$

The linearizing transformations used in this case are

$$
t=x, \quad v=y^{2}-z^{2}, \quad w=2 y z .
$$

The solution of (23) is given by

$$
v=c_{1} t^{2}+c_{2} t+c_{3}, \quad w=c_{4} t^{2}+c_{5} t+c_{6},
$$

where all $c_{1} \ldots, c_{6}$ are arbitrary constants. By using the transformation (24), we get the general solution of (22) in the implicit form

$$
y^{2}-z^{2}=c_{1} x^{2}+c_{2} x+c_{3}, \quad 2 y z=c_{4} x^{2}+c_{5} x+c_{6} .
$$

It leads us to general solution in the explicit form

$$
\begin{aligned}
& y=\frac{ \pm 1}{\sqrt{2}} \sqrt{\sqrt{\left(c_{1} x^{2}+c_{2} x+c_{3}\right)^{2}+\left(c_{4} x^{2}+c_{5} x+c_{6}\right)^{2}}+\left(c_{1} x^{2}+c_{2} x+c_{3}\right)}, \\
& z=\frac{ \pm 1}{\sqrt{2}} \sqrt{\sqrt{\left(c_{1} x^{2}+c_{2} x+c_{3}\right)^{2}+\left(c_{4} x^{2}+c_{5} x+c_{6}\right)^{2}}-\left(c_{1} x^{2}+c_{2} x+c_{3}\right)} .
\end{aligned}
$$

Example 3 Consider the nonlinear system of third-order ODEs

$$
\begin{aligned}
& y^{\prime \prime \prime}+\left(\frac{3 y y^{\prime}}{y^{2}+z^{2}}+\frac{3 z z^{\prime}}{y^{2}+z^{2}}-3\right) y^{\prime \prime}-\left(-\frac{3 z y^{\prime}}{y^{2}+z^{2}}+\frac{3 y z^{\prime}}{y^{2}+z^{2}}\right) z^{\prime \prime} \\
& -\frac{3 y z^{\prime 2}}{y^{2}+z^{2}}-\frac{6 z y^{\prime} z^{\prime}}{y^{2}+z^{2}}+\frac{3 y z^{\prime 2}}{y^{2}+z^{2}}+2 y^{\prime}+3 y=0, \\
& z^{\prime \prime \prime}+\left(-\frac{3 z y^{\prime}}{y^{2}+z^{2}}+\frac{3 y z^{\prime}}{y^{2}+z^{2}}\right) y^{\prime \prime}-\left(\frac{3 y z^{\prime}}{y^{2}+z^{2}}+\frac{3 z z^{\prime}}{y^{2}+z^{2}}-3\right) z^{\prime \prime} \\
& +\frac{3 z y^{\prime 2}}{y^{2}+z^{2}}-\frac{6 y y^{\prime} z^{\prime}}{y^{2}+z^{2}}-\frac{3 z z^{\prime 2}}{y^{2}+z^{2}}+2 z^{\prime}+3 z=0 .
\end{aligned}
$$

The coefficients

$$
\begin{aligned}
& \alpha_{1}=\frac{3 y}{y^{2}+z^{2}}, \quad \alpha_{0}=-3, \quad \beta_{1}=\frac{-3 z}{y^{2}+z^{2}}, \quad \gamma_{2}=\frac{-3 y}{y^{2}+z^{2}}, \\
& \gamma_{1}=2, \quad \gamma_{0}=3 y, \quad \delta_{2}=\frac{3 y}{y^{2}+z^{2}}, \quad \delta_{0}=3 z, \quad \gamma_{3}=\delta_{3}=\delta_{1}=\beta_{0}=0 .
\end{aligned}
$$

satisfy the CR equations and (18). Hence, (25) is transformable to a linear system of third-order ODEs. It linearizes to

$$
v^{\prime \prime \prime}+\frac{6}{t^{3}} v=0, \quad w^{\prime \prime \prime}+\frac{6}{t^{3}} w=0,
$$

where prime denotes differentiation with respect to $t$. The linearizing transformations which establish the above correspondence between the nonlinear and linear systems are

$$
t=e^{x}, \quad v=y^{2}-z^{2}, \quad w=2 y z
$$

The solution of (27) is 


$$
\begin{aligned}
& v=c_{1} t^{-1}+t^{2}\left\{c_{2} \cos (\sqrt{2} \ln t)+c_{3} \sin (\sqrt{2} \ln t)\right\}, \\
& w=c_{4} t^{-1}+t^{2}\left\{c_{5} \cos (\sqrt{2} \ln t)+c_{6} \sin (\sqrt{2} \ln t)\right\},
\end{aligned}
$$

where all $c_{1} \ldots, c_{6}$ are arbitrary constants. By employing transformation (28), the general solution of (25) is given explicitly by

$$
\begin{aligned}
y=\frac{ \pm 1}{\sqrt{2}} & {\left[\sqrt{\left[c_{1} e^{-x}+e^{2 x}\left\{c_{2} \cos (\sqrt{2} x)+c_{3} \sin (\sqrt{2} x)\right\}\right]^{2}+\left[c_{4} e^{-x}+e^{2 x}\left\{c_{5} \cos (\sqrt{2} x)+c_{6} \sin (\sqrt{2} x)\right\}\right]^{2}}\right.} \\
& \left.+c_{1} e^{-x}+e^{2 x}\left\{c_{2} \cos (\sqrt{2} x)+c_{3} \sin (\sqrt{2} x)\right\}\right]^{\frac{1}{2}}, \\
z=\frac{ \pm 1}{\sqrt{2}} & {\left[\sqrt{\left[c_{1} e^{-x}+e^{2 x}\left\{c_{2} \cos (\sqrt{2} x)+c_{3} \sin (\sqrt{2} x)\right\}\right]^{2}+\left[c_{4} e^{-x}+e^{2 x}\left\{c_{5} \cos (\sqrt{2} x)+c_{6} \sin (\sqrt{2} x)\right\}\right]^{2}}\right.} \\
& \left.-c_{1} e^{-x}-e^{2 x}\left\{c_{2} \cos (\sqrt{2} x)+c_{3} \sin (\sqrt{2} x)\right\}\right]^{\frac{1}{2}} .
\end{aligned}
$$

Example 4 The nonlinear system of ODEs

$$
\begin{aligned}
& y^{\prime \prime \prime}+3\left(1+y^{\prime}\right) y^{\prime \prime}-z^{\prime} z^{\prime \prime}+y^{\prime 3}-3 y^{\prime} z^{2}+3 y^{\prime 2}-3 z^{\prime 2}+3 y^{\prime}+(1+x)=0, \\
& z^{\prime \prime \prime}+3\left(1+y^{\prime}\right) z^{\prime \prime}+3 z^{\prime} y^{\prime \prime}+z^{\prime 3}+3 y^{\prime 2} z^{\prime}+6 y^{\prime} z^{\prime}+3 z^{\prime}=0,
\end{aligned}
$$

satisfies conditions of Theorems 1 and 2, which guarantee its transformation to a linear form. The linearizing transformation is

$$
t=x, \quad y=\frac{1}{2} \ln \left(v^{2}+w^{2}\right)-x, \quad z=\arctan \left(\frac{w}{v}\right),
$$

that brings the nonlinear system to a linear form

$$
v^{\prime \prime \prime}+t v=0, \quad w^{\prime \prime \prime}+t w=0 .
$$

\section{Conclusion}

We have used CSA to linearize a class of two-dimensional systems of third-order ODEs which can be obtained from a scalar third-order Lie linearizable ODE. This class has a CR structure. The linearization is achieved fairly trivially this way. Trying to linearize without recourse to CSA, the process is much more difficult. It would be worth exploring the case of systems of non-linearizable third-order ODEs corresponding to a linearizable third-order scalar ODE. If it behaves in a way similar to the second-order systems of this type, it should lead to cases where the system has no symmetry but the solution is obtainable by "complex linearization". It would also be worth while to extend this analysis to fourth and higher orders.

Acknowledgements Two of us (HMD and AQ) are grateful to the Higher Education Commission of Pakistan for support under their Project No. 3054.

Open Access This article is distributed under the terms of the Creative Commons Attribution 4.0 International License (http:// creativecommons.org/licenses/by/4.0/), which permits unrestricted use, distribution, and reproduction in any medium, provided you give appropriate credit to the original author(s) and the source, provide a link to the Creative Commons license, and indicate if changes were made.

\section{References}

1. Ali, S.: Complex Symmetry Analysis. PhD Thesis, National University of Sciences and Technology (2008)

2. Ali, S.; Mahomed, F.M.; Qadir, A.: Linearization criteria for systems of two second order differential equations by complex methods. Nonlinear Dyn. 66, 77 (2011)

3. Ali, S.; Safdar, M.; Qadir, A.: Linearization from complex Lie point transformations. J. Appl. Math. 2014, 793247 (2014)

4. Bagderina, Y.Y.: Linearization criteria for a system of two second order ordinary differential equations. J. Phys. A 46, 465201 (2010)

5. Chern, S.S.: The geometry of the differential equation $y^{\prime \prime \prime \prime}=F\left(x, y, y^{\prime \prime}, y^{\prime \prime \prime}\right)$. Tensor N.S. 28, 173 (1940) 
6. Dutt, H.M.; Safdar, M.: Linearization of two dimensional complex-linearizable systems of second order ordinary differential equations. Appl. Math. Sci. 9, 2889 (2015)

7. Grebot, G.: The characterization of third order ordinary differential equations admitting a transitive fibre-preserving point symmetry group. J. Math. Anal. Appl. 206, 364 (1997)

8. Ibragimov, N.H.; Meleshko, S.V.: Linearization of third order ordinary differential equations by point and contact transformations. J. Math. Anal. Appl. 308, 266 (2005)

9. Ibragimov, N.H.; Meleshko, S.V.; Suksern, S.: Linearization of fourth-order ordinary differential equations by point transformations. J. Phys. A Math. Theor. 41, 235206 (2008)

10. Lie, S.: Theorie der transformationsgruppen. Math. Ann. 16, 441 (1880)

11. Lie, S.: Klassifikation und integration von gewönlichen differentialgleichungenzwischen $x, y$, die eine Gruppe von transformationen gestaten. Arch. Math. VIII, IX, 187 (1883)

12. Mahomed, F.M.; Leach, P.G.L.: Symmetry Lie algebras of $n$th order ordinary differential equations. J. Math. Anal. Appl. 151, $80(1990)$

13. Mahomed, F.M.: Point symmetry group classification of ordinary differential equation: a survey of some results. Math. Methods Appl. Sci. 30, 1995 (2007)

14. Mahomed, F.M.; Qadir, A.: Linearization criteria for a system of second-order quadratically semi-linear ordinary differential equations. Nonlinear Dyn. 48, 417 (2007)

15. Mahomed, F.M.; Qadir, A.: Invariant linearization criteria for systems of cubically semi-linear second order ordinary differential equations. J. Nonlinear Math. Phys. 16, 1 (2009)

16. Neut, S.; Petitot, M.: La géométrie de l'équation $y^{\prime \prime \prime}=f\left(x, y, y^{\prime}, y^{\prime \prime}\right)$. C. R. Acad. Sci. Paris Sér I(335), 515 (2002)

17. Sookmee, S.; Meleshko, S.V.: Linearization of two second-order ordinary differential equations via fibre preserving point transformations. ISRN Math. Anal. 2011, 452689 (2001)

18. Sookmee, S.; Meleshko, S.V.: Conditions for linearization of a projectable system of two second order ordinary differential equations. J. Phys. A 41, 402001 (2008)

19. Suksern, S.; Meleshko, S.V.; Ibragimov, N.H.: Criteria for fourth order ordinary differential equations to be linearizable by contact transformations. Commun. Nonlinear Sci. Numer. Simul. 14, 2619 (2009)

20. Tressé, A.: Sur les Invariants differentiels des groupes continus de transformations. Acta. Math. 18, 1 (1894)

21. Wafo Soh, C.; Mahomed, F.M.: Linearization criteria for a system of second order ordinary differential equations. Int. J. Nonlinear Mech. 36, 671 (2001)

Publisher's Note Springer Nature remains neutral with regard to jurisdictional claims in published maps and institutional affiliations. 